\title{
Femtosecond Coherent Anti-Stokes Raman Spectroscopy (fs-CARS) with Nitrobenzene and Nitomethane
}

\author{
VINU V NAMBOODIRI ${ }^{1}$, MOHAMMED AHMED ${ }^{1}$, G KRISHNA PODAGATLAPALLI ${ }^{2}$ and AJAY K \\ SINGH $^{1 *}$ \\ ${ }^{1}$ Radiation and Photochemistry Division, Bhabha Atomic Research Centre, Mumbai 400 085, India \\ ${ }^{2}$ Advanced Centre of Research in High Energy Materials, University of Hyderabad, Hyderabad 500 \\ 046, India
}

(Received on 30 November 2014; Revised on 19 December 2014; Accepted on 5 January 2015)

\begin{abstract}
Time-resolved coherent anti-Stokes Raman Scattering (CARS) is a nonlinear spectroscopic tool, which has gained importance with the development of ultrafast lasers due to its ability to probe ultrafast dynamical processes in molecules. Apart from the time resolution, the superiority of CARS over spontaneous Raman scattering derives from its high signal generation ability and coherent, laser like, signal emission. In this contribution, we describe the development of an experimental facility for the time-resolved coherent anti-Stokes Raman scattering spectroscopy and its application to study the vibrational dynamics of molecules containing nitro $\left(\mathrm{NO}_{2}\right)$ groups. fs-CARS experiments were carried out in neat liquids of nitrobenzene, nitromethane and their mixture. A detailed mapping of the dynamics initiated by the stimulated Raman process is achieved from the spectrum of the transient CARS signal. The CARS transient shows rich beating structure corresponding to frequencies as high as $10 \mathrm{THz}$. The Fourier transform of the transient allows accurate determination of the vibrational energy differences. This methodology is capable of providing the dephasing behaviour and spectral information of the molecular system at the same time.The results demonstrate that CARS spectroscopy with femtosecond time resolution and wavenumber-resolved detection is a powerful tool for the characterization of dynamics in the electronic ground state of molecules.
\end{abstract}

Key Words: Femtosecond; CARS; Vibrational Dynamics; Nitromethane; Nitrobenzene

\section{Introduction}

With the application of lasers, spontaneous Raman spectroscopy developed as a powerful analytical tool and is applied in a wide variety of areas. However, due to inherently weak signal levels and fluorescence background, its applicability is restricted when the sample concentration is too low or the sample is fluorescent. These difficulties associated with spontaneous Raman spectroscopy can be overcome by coherent Raman spectroscopy (Potma and Mukamel, 2013). Coherent Raman techniques are non-linear spectroscopic techniques and provide much higher signal levels compared to spontaneous Raman signals. While spontaneous Raman signal is emitted in all directions, the coherent Raman signal is a laser like emission in a well-defined direction, which makes the detection easier and background-free. Coherent Raman spectroscopic techniques are now widely being used as an alternative to conventional Raman spectroscopy due to its high signal levels and coherent nature of the signal.

Coherent anti-Stokes Raman scattering (CARS) (Potma and Mukamel, 2013; El-Diasty, 2011) is one of the coherent Raman techniques that has evolved as a versatile technique for material characterisation and imaging in various areas of science. Though

\footnotetext{
*Author for Correspondence: E-mail: aksingh@barc.gov.in; Tel: 022-25596116
} 
experimentally complex compared to conventional Raman, the high signal levels and natural rejection of fluorescence eliminates the most common factors plaguing the applicability of conventional Raman spectroscopy. Frequency domain CARS, using narrow band lasers, has proved to be an efficient vibrational spectroscopic tool in low-pressure gases, combustion processes, plasma and also in molecular spectroscopy and microscopy (Djaker et al., 2007; Roy et al., 2010).

The development of ultrafast lasers added another dimensionality to laser spectroscopy. Timeresolved spectroscopy using ultrashort laser pulses of pico/femtosecond duration made it possible to probe fast molecular dynamical processes in real time. The time scales provided by ultrafast lasers correspond to the vibrational period of molecular vibrations (100 fs $\sim 150 \mathrm{~cm}^{-1}$ ) and are ideal for time-resolved probing of vibrational dynamics in molecules. Most of the femtosecond time-resolved studies have been performed on investigation of dynamics of molecular systems in electronically excited states. Femtosecond time-resolved CARS (fs-CARS) is an experimental technique, which is capable of studying dynamics in the electronic ground state of molecular systems. It was first reported by probing ultrafast vibrational dynamics in several liquids (Leonhardt et al., 1987). Using the broad spectral width of femtosecond pulses, several vibrational modes can be excited coherently (wave packets) and can monitor their relaxation processes in real time. As a result of the intense signal, sensitivity and inherent florescence discrimination, CARS is of considerable interest in time-resolved spectroscopy. Some of the applications of timeresolved CARS include studies of vibrational dephasing times in solution, collisional relaxation times, and study of the excited state properties (Schmitt et al., 1998; Vierheilig et al., 1999).The large spectral width of fs pulses limits the spectral resolution achieved by fs-CARS. Developments in pulse shaping technology (Weiner, 2011; Monmayrant et al., 2010) provide a way to improve the spectral resolution in fs-CARS.

In this contribution, we describe the development of an experimental facility for time-resolved CARS using femtosecond laser pulses and its application in probing time-resolved vibrational dynamics in nitrobenzene, nitromethane and their mixture. Many of the high-energy materials contain nitro groups and the aim of the CARS experiment was to explore the applicability of CARS in deciphering the electronic ground state vibrational dynamics of high energy materials. The paper is organised as follows: next section provides a brief account of the theory of CARS followed by the experimental description. The final section discusses the experimental results on nitromethane, nitrobenzene and their mixture.

\section{Theory}

The theory of time-resolved Four-Wave-Mixing (FWM) processes can be formulated based on third order perturbation theory, which is used to calculate the third order polarization, $P^{(3)}(t)$ responsible for the signal generation (Mukamel, 1995; Tannor, 2007). In the semiclassical picture presented here, the electromagnetic field is treated classically and the matter is described quantum mechanically using the wave function approach. The fundamental observable in CARS is the third order polarisation that is generated by the interaction of three laser pulses namely the pump $E_{p u}\left(\omega_{p u}, k_{p u}\right)$, Stokes $E_{s}\left(\omega_{s}, k_{s}\right)$ and the probe $E_{p r}\left(\omega_{p r}, k_{p r}\right)$ pulses. The time dependent polarisation $P(t)$ induced in a system with a state vector $\psi(t)$ is given by

$$
P(t)=\langle\psi(t)|\mu| \psi(t)\rangle
$$

where $\mu$ is the dipole operator. Inorder to calculate the third order polarisation, the perturbative expansion of the wave function is used.

$$
|\psi(t)\rangle=\sum_{N=0}^{\infty}\left|\psi^{(N)}(t)\right\rangle
$$

wave functions of different orders can be obtained iteratively as

$$
\left|\psi^{(N)}(t)\right\rangle=-\frac{i}{\hbar} \int_{-\infty}^{t} e^{\frac{i H\left(t-t^{\prime}\right)}{\hbar}} H_{i n t}\left(t^{\prime}\right)\left|\psi^{(N-1)}\left(t^{\prime}\right)\right\rangle d t^{\prime}
$$

Through successive interactions with the three pulses of the CARS process, the wave function acquires spatial dependencies associated with each of the pulses (Faeder et al., 2001). For higher order 
wave functions, the order of the wave vectors denotes the time ordering of the interactions with the fields. For example, the third order wave function relevant for CARS is $\left|\psi_{k_{p u}-k_{S}+k_{p r}}^{(3)}(t)\right\rangle$ which is created by the absorption of a pump photon with wave vector $k_{p u}$, stimulated emission of a photon with wave vector $k_{s}$ followed by the absorption of a photon with wave vector $k_{p r}$. This sequence of events in CARS can be pictorially represented using double-sided Feynman diagrams. The Feynman diagram relevant for CARS and the corresponding energy level diagram are shown in Fig. 1.

The third order polarisation responsible for the CARS signal generation can be written as

$$
P^{(3)}(t)=\left\langle\psi^{(0)}(t)|\mu| \psi_{k_{p u}-k_{S}+k_{p r}}^{(3)}(t)\right\rangle+c . c
$$

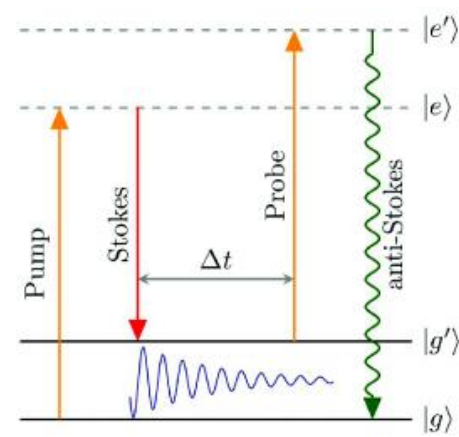

A

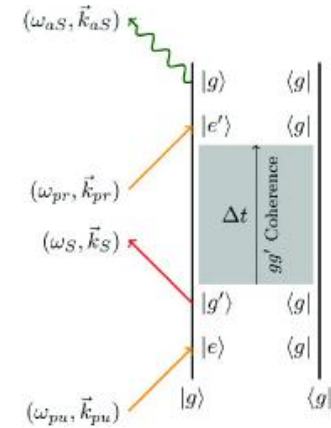

B
Fig. 1: Energy level diagram (A) and double sided Feynman diagram (B) corresponding to CARS process

The third order wave function $\left|\psi_{k_{p u}-k_{S}+k_{p r}}^{(3)}(t)\right\rangle$ can be written, assuming the energy level diagram given in Fig. 1 as

$$
\begin{gathered}
\psi^{(3)}(t)=\left(-\frac{i}{\hbar}\right)^{3} \int_{0}^{t} \int_{0}^{t_{p r}} \int_{0}^{t_{S}} e^{-\frac{i}{\hbar} H_{e^{\prime}}\left(t-t_{p r}\right)} \\
{\left[-\mu E_{p r}\left(t_{p r}\right)\right] e^{-\frac{i}{\hbar} H_{g^{\prime}}\left(t_{p r}-t_{S}\right)}} \\
{\left[-\mu E_{S}\left(t_{S}\right)\right] e^{-\frac{i}{\hbar} H_{e}\left(t_{S}-t_{p u}\right)}\left[-\mu E_{p u}\left(t_{p u}\right)\right] e^{-\frac{i}{-} H_{g} t_{p u}}} \\
\psi_{g}(0) d t_{p u} d t_{S} d t_{p r}
\end{gathered}
$$

This equation can be simply interpreted as the evolution of the ground state wave function $\psi_{g}(0)$ under the influence of the ground state Hamiltonian $H_{g}$ till the interaction of the first pump pulse $E_{p u}\left(t_{p u}\right)$ at time $t_{p u}$. The interaction of the pump pulse creates an excited wave function $\left[-\mu E_{p u}\left(t_{p u}\right)\right] e^{-\frac{i}{\hbar} H_{g} t_{p u}} \psi_{g}(0)$ which is nothing but the ground state wave function scaled by $\left[-\mu E_{p u}\left(t_{p u}\right)\right]$. This newly created excited wave packet then evolves under the excited state Hamiltonian $H_{e}$ till the interaction with the Stokes pulse $E_{s}\left(t_{s}\right)$ at time $t_{s}$. This interaction causes a stimulated emission to an excited vibrational level $g^{\prime}$ and the new wave function generated by the stimulated emission then evolves under the $H_{g}$, Hamiltonian, till the interaction with the probe pulse $E_{p r}\left(t_{p r}\right)$ at time $t_{p r}$. The interaction with the probe pulse promotes the wave packet to the excited state, which then evolves under the excited state Hamiltonian $H_{e^{\text {, }}}$ till the emission of the anti-Stokes field.

In order to calculate the intensity of the antiStokes emission, the third order polarisation given by equation (4) can be converted to the frequency domain (Fourier transform) and used as the source term in the Maxwell's equation.

$$
\left(\nabla^{2}+\frac{\omega^{2}}{c^{2}} \varepsilon(\omega)\right) E=-\frac{4 \pi \omega^{2}}{c^{2}} P^{3}(\omega)
$$

The solution of this equation gives the signal intensity as

$$
I_{\text {CARS }} \propto\left|\chi^{(3)}\right|^{2} I_{p u} I_{S} I_{p r} l^{2}\left(\frac{\sin \frac{\Delta k l}{2}}{\frac{\Delta k l}{2}}\right)^{2}
$$

Where, $\chi^{(3)}$ is the third order susceptibility, $I_{i}(i$ $=p u, S, p r)$ are the intensities of the three input pulses and $l$ is the interaction length. $\Delta k$ is the phase mismatch which is given by

$$
\Delta k=k_{p u}-k_{S}+k_{p r}-k_{a S}
$$

The phase mismatch is the wave vector mismatch between the three input fields and the 
emitted signal. The sinc function dependence of the signal intensity on the phase mismatch suggests that the signal rapidly decreases to zero with increasing phase mismatch. Thus, for efficient CARS signal generation proper phase matching should be ensured. This is achieved by using a special beam geometry, which is described in the next section.

\section{Experimental Setup for Time-Resolved CARS}

Femtosecond laser pulses required for the experiment are generated by a commercial laser system $(3 \mathrm{~mJ}, 1$ $\mathrm{kHz}$, Amplitude Technologies, Trident-M) delivering pulses of duration $\sim 50 f s$ with central wavelength $800 \mathrm{~nm}$. Two multipass optical parametric amplifiers (OPA; Light Conversion, TOPAS-C) are used in order to generate the different wavelengths required for the CARS experiment.Each of the OPA is pumped by $1 \mathrm{~mJ}$ of $800 \mathrm{~nm}$ output from the Trident-M system. One of the OPA output is continuously tunable from $450 \mathrm{~nm}$ to $10000 \mathrm{~nm}$ and the other from $250 \mathrm{~nm}$ to $2700 \mathrm{~nm}$. The pulse characteristics are determined from autocorrelation and FROG traces (Pulse Check, APE). The pump and probe pulses were of the same wavelength and derived from one of the OPA output using a 50/50 beam splitter.

The pump and Stokes wavelengths were 590 $\mathrm{nm}$ and $635 \mathrm{~nm}$ respectively and correspond to a wavenumber difference of $1200 \mathrm{~cm}^{-1}$. All the three laser pulse energies were fixed to be around 25-30 $n J$ using neutral density filters. The broad spectral width of the pulses enabled excitation of Raman modes within a region of $+400 \mathrm{~cm}^{-1}$ from the centre of excitation. For an efficient CARS signal generation, perfect overlap of the input pulses has to be ensured both spatially and temporally. A schematic of the complete experimental setup for CARS is shown in Fig. 2. Spatial overlap of the pulses is necessary to achieve phase matching. The phase matching is ensured by using the Folded-BOXCAR arrangement of the input beams. In this arrangement, the three beams involved in the CARS process, pump, probe and Stokes are aligned parallel to each other and are made to pass through three corners of a rectangular box with the help of a mask as detailed in Fig. 3. The selection of wavelength was based on the nitro group vibration characteristics.
The three beams were then focused onto the sample using a lens of $10 \mathrm{~cm}$ focal length. The time delay between the pulses was adjusted using computer controlled linear translation stages (Newport ILS 150) mounted with retroreflecting mirrors. The pump and Stokes pulses for the CARS process were time coincident and the timing of the probe pulse was variable. The spatially separated CARS signal from the sample is collimated using another lens $(\mathrm{f}=10 \mathrm{~cm})$ and sent to a spectrometer (ANDOR Shamrock) attached with a TE cooled CCD (ANDOR Newton). Neat liquids of nitromethane and nitrobenzene were

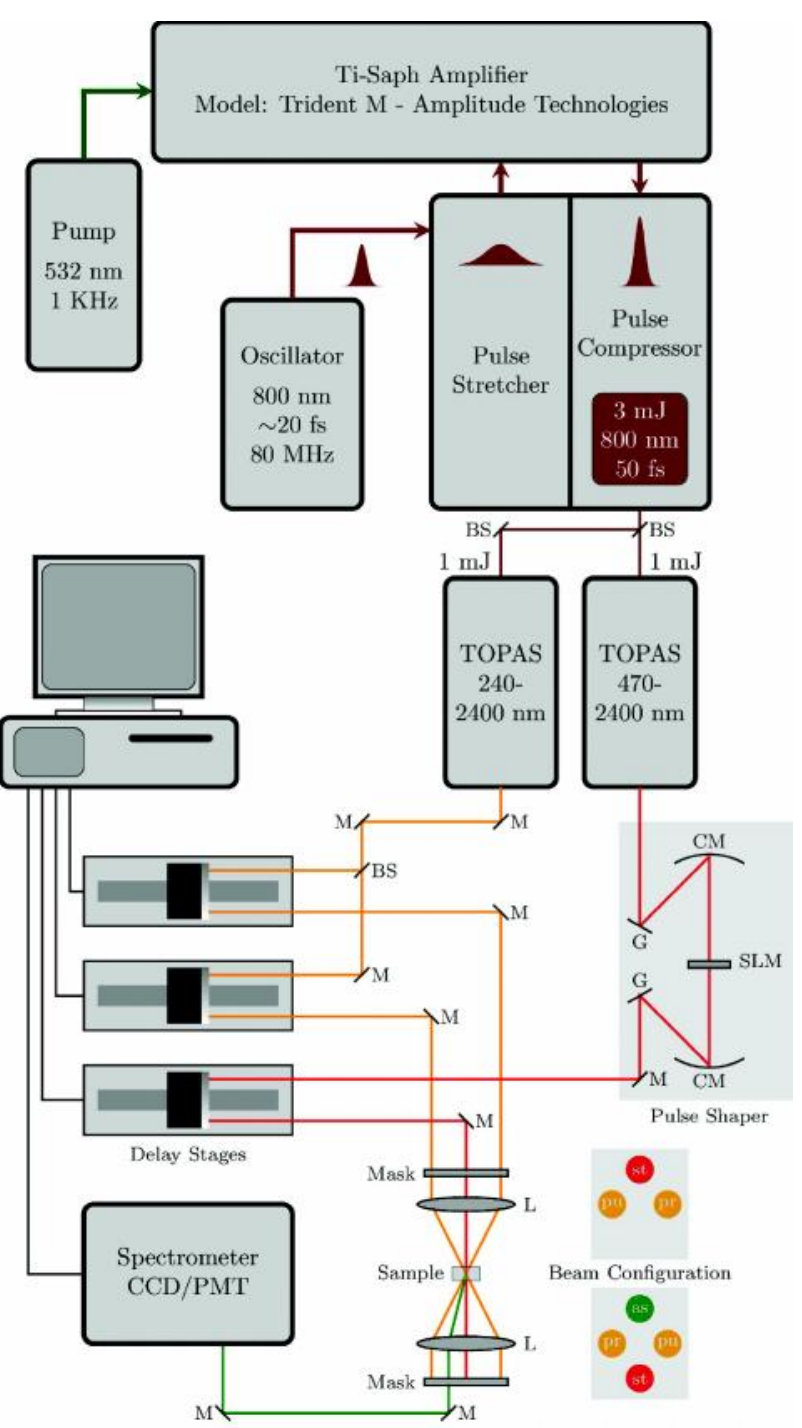

Fig. 2: Experimental setup for CARS using folded BOXCAR beam geometry: M-Mirror, BS- Beam Splitter, GGrating, and L-Lens. The delay stages are linear translation stages fitted with a retroreflector 


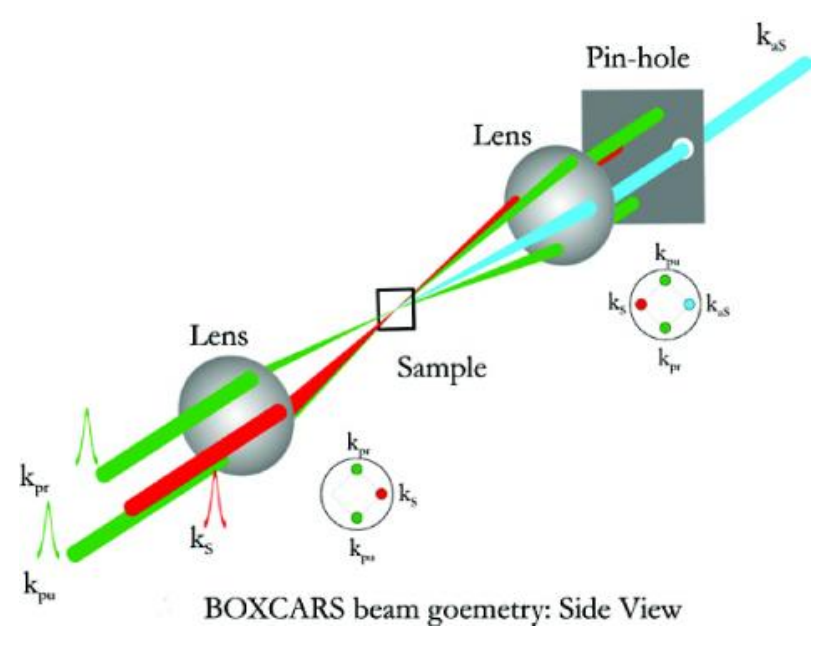

Fig. 3: Folded BOXCARS beam arrangement

used for the experiments.

\section{Results and Discussion}

In the following, results from the CARS spectrum obtained from neat liquids of nitromethane, nitrobenzene and their mixture are discussed. These molecules do not absorb in the visible spectral region, therefore the CARS process here does not involve any excited electronic state.

\section{CARS on Nitrobenzene}

The time and frequency-resolved CARS transient of nitrobenzene is shown in Fig. 4. The transient shows well defined beating structure arising due to the simultaneous excitation of different vibrational modes, which fall within spectral width of the excitation pulses. A beating structure is observed in the transient CARS signal. Such beating structures are well known and are due to quantum beating of the different coherently excited vibrational modes of the molecules.

To make these signal modulations more visible, Fig. 5A presents a frequency slice of the transient. An analysis of the beating frequency can be performed by fast Fourier transformation (FFT) and the frequency difference between the beating vibrational modes is shown in Fig. 5B. The peak location along the FFT wavenumber axis corresponds to the wavenumber differences of two coherently excited vibrational modes of nitrobenzene. It is now obvious

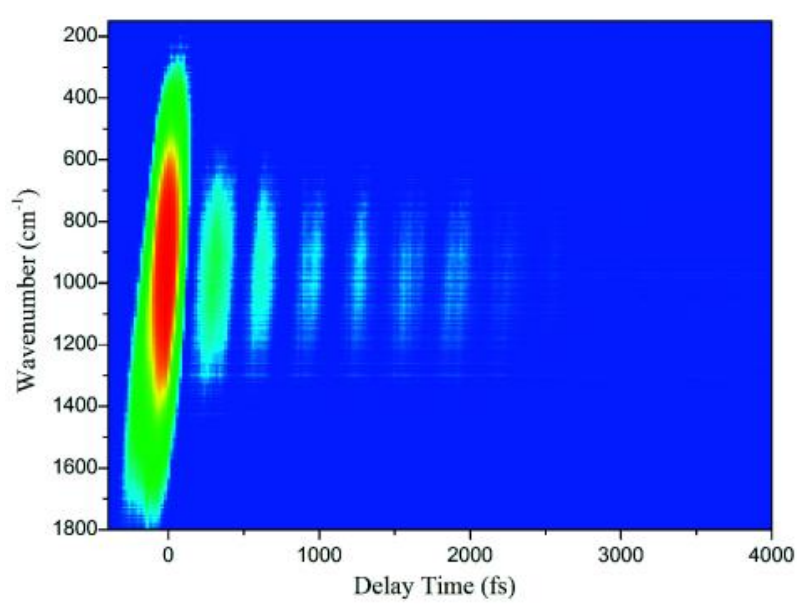

Fig. 4: Time and frequency resolved CARS spectrum of nitrobenzene

that the complex oscillatory pattern of the time domain signal is mainly attributable to four peaks.

An assignment of the beating wavenumbers is possible taking into account the Raman modes excited by the femtosecond pump and Stokes pulses. It is now appropriate to compare the obtained results with spontaneous Raman spectrum of nitrobenzene (Fig. $6)$.

The beat frequencies seen in the Fourier spectrum shows peaks at wavenumber positions 20 $\mathrm{cm}^{-1}, 103 \mathrm{~cm}^{-1}, 238 \mathrm{~cm}^{-1}$ and $337 \mathrm{~cm}^{-1}$. These peaks correspond to the frequency difference between the $853 \mathrm{~cm}^{-1}, 1003 \mathrm{~cm}^{-1}, 1022 \mathrm{~cm}^{-1}, 1107 \mathrm{~cm}^{-1}$ and 1347 $\mathrm{cm}^{-1}$ vibrational modes of nitrobenzene. The highest beat frequency of $337 \mathrm{~cm}^{-1}$ between the nitrobenzene vibrational modes at $1003 \mathrm{~cm}^{-1}$ and $1347 \mathrm{~cm}^{-1}$ corresponds to a frequency of $10.1 \mathrm{THz}$. This is indicative of the high temporal resolution of the experiment, which enables probing of terahertz phenomena with appreciable accuracy.

\section{CARS on Nitromethane and Mixture}

Similar experiments were carried out in nitromethane and a mixture of nitromethane and nitrobenzene (1:1 $\mathrm{v} / \mathrm{v})$. The nitromethane CARS transient is dominated by a single Raman mode at $917 \mathrm{~cm}^{-1}$ and hence did not show any beating structure (Fig. 7B). In the nitromethane case, no FFT analysis was necessary as there was only one prominent Raman mode and 


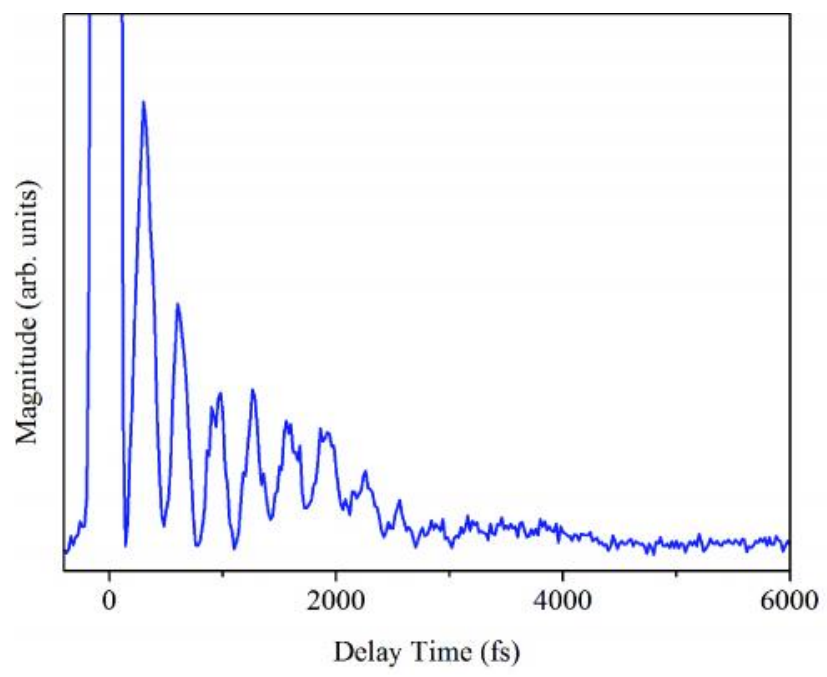

A

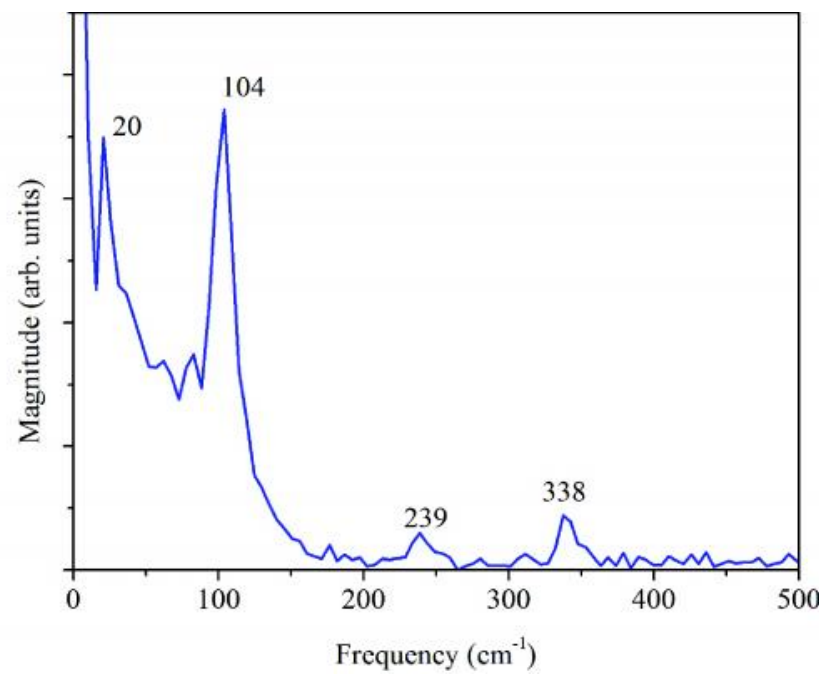

B

Fig. 5: A: Time-resolved CARS transient showing rapid modulations (spectral slice). B: FFT corresponding to the transient showing the beat frequencies

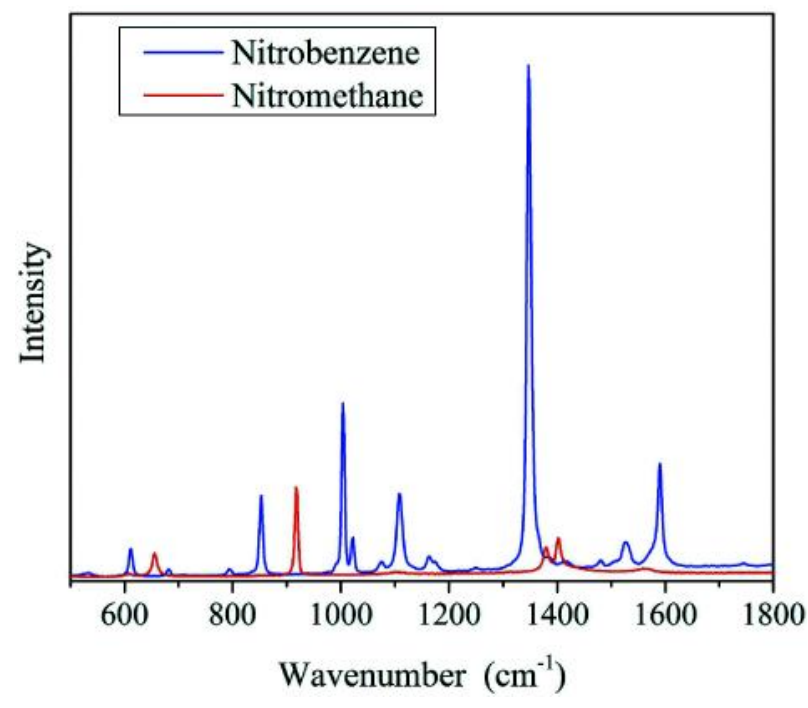

Fig. 6: Spontaneous Raman spectrum of nitrobenzene (blue) and nitromethane (red) displaying the vibrational modes excited by the femtosecond pump and Stokes pulses

the CARS signal decays exponentially. The decay time $\tau=T_{2} / 2$ is $\approx 1139$ fs (Shkurinov, 1994).

The CARS transient of the mixture showed modulations of the CARS signal (Fig. 7A) similar to the case of nitrobenzene. Fourier analysis of the CARS transient of the mixture showed an additional beat frequency component at $86 \mathrm{~cm}^{-1}$ (Fig. 8) which arises from the beating between the vibrational modes of nitromethane $\left(917 \mathrm{~cm}^{-1}\right)$ and nitrobenzene $(1003$ $\mathrm{cm}^{-1}$ ). The presence of additional frequency components in the CARS transient can be used as a signature to identify components in a mixture. Further analysis is being carried out to extract the dephasing time of different participating vibrational modes and to analyse how the dephasing times are influenced in a mixture.

\section{Conclusions}

An experimental facility for time-resolved CARS using femtosecond laser pulses has been described with two examples of organic nitro compounds. Spectrally dispersed CARS signal leads to a twodimensional (time and wavenumber) data set. An analysis of this two-dimensional time domain data on the basis of Fourier transform methods enables one to obtain detailed spectral information on the vibrational mode structure in addition to the vibrational dephasing times that are readily deducible from the decay behaviour of the transient signals. It has been demonstrated that CARS spectroscopy with femtosecond time resolution and wavenumber resolved detection is a powerful tool for the characterization of dynamics in the electronic ground state of molecules. 


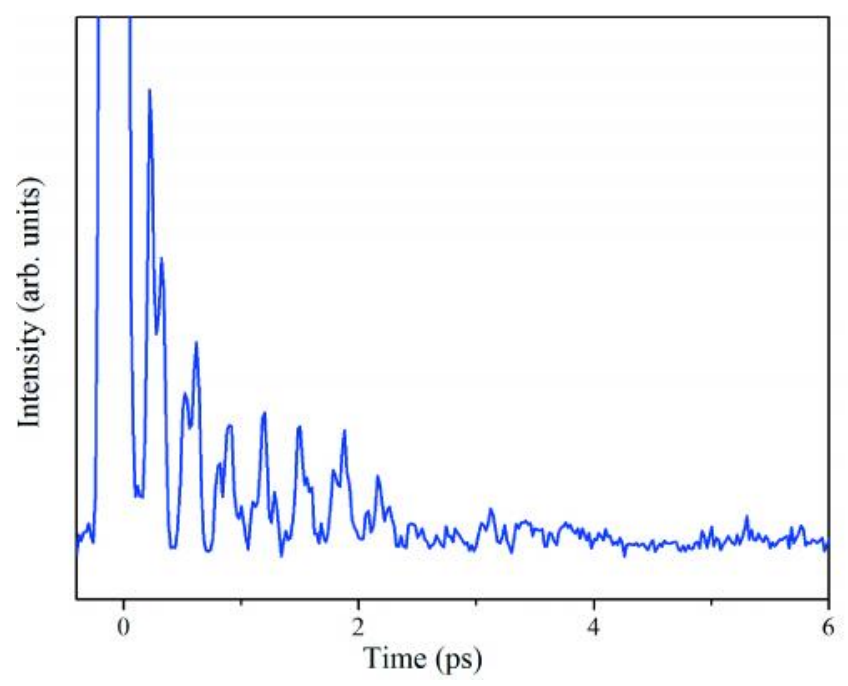

A

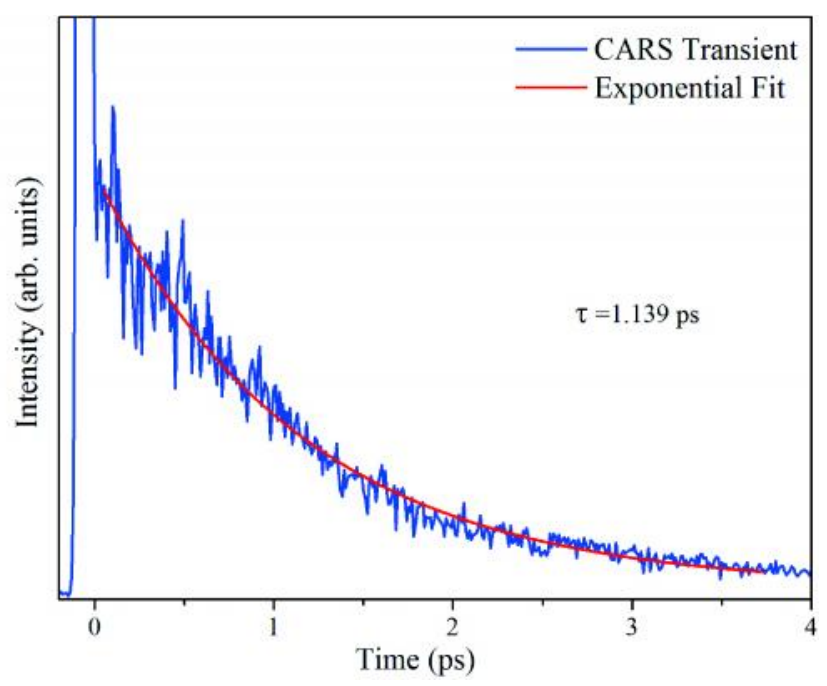

B

Fig. 7: A: CARS transient from 1:1 (by volume) mixture of nitromethane and nitrobenzene. B: CARS transient from pure nitromethane

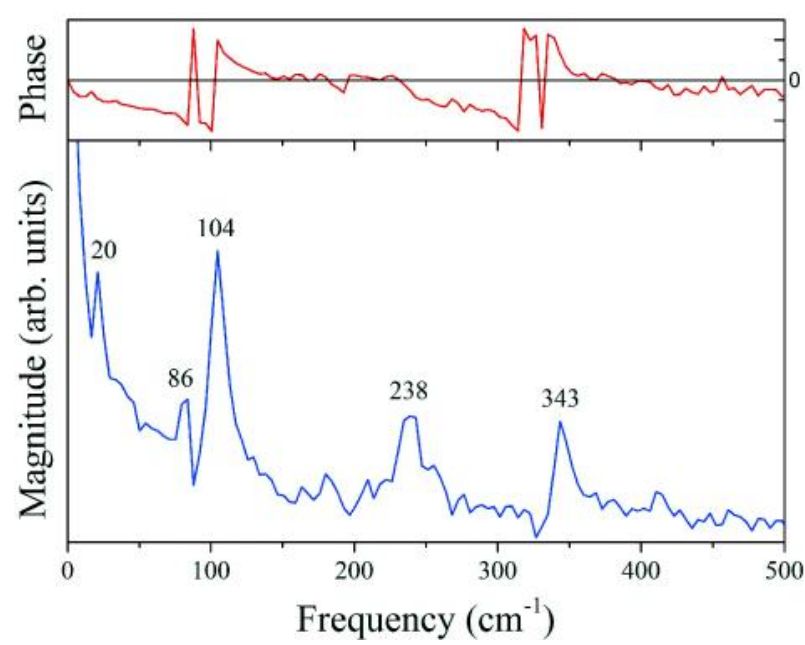

Fig. 8: FFT of the CARS transient of mixture

\section{References}

Brown E, Zhang Q and Dantus M (1999) Femtosecond transientgrating techniques: Population and coherence dynamics involving ground and excited states $J$ of Chemical Physics $1105772-5788$

Djaker N, Lenne P, Marguet D, Colonna A, Hadjur C and Rigneault H (2007) Coherent anti-Stokes Raman scattering microscopy (CARS): Instrumentation and applications Nuclear Instruments and Methods in Physics Research A
$571177-181$

El-Diasty F (2011) Coherent anti-Stokes Raman scattering: Spectroscopy and microscopy Vibrational Spectroscopy $\mathbf{5 5} 1-37$

Faeder J, Pinkas I, Knopp G, Prior Y and Tannor D J (2001) Vibrational polarization beats in femtosecond coherent antiStokes Raman spectroscopy: A signature of dissociative pump-dump-pump wave packet dynamics $J$ Chemical Physics 115 8440-8454

Leonhardt R, Holzapfel W, Zinth W and Kaiser W (1987) Terahertz quantum beats in molecular liquids Chemical Physics Letters 133 373-377

Monmayrant A, Weber S and Chatel B (2010) A newcomer's guide to ultrashort pulse shaping and characterization $J$ Physics B: Atomic Molecular and Optical Physics 43 103001

Mukamel S (1995) Principles of Nonlinear Optical Spectroscopy Oxford University Press New York

Potma E and Mukamel S (2013) Theory of Coherent Raman Scattering. In: Coherent Raman Scattering Microscopy (Eds: Cheng J and Xie X S) pp 3-42 CRC Press Taylor \& Francis Group

Roy S, Gord J R and Patnaik A K (2010) Recent advances in coherent anti-Stokes Raman scattering spectroscopy: Fundamental developments and applications in reacting flows Progress in Energy and Combustion Science 36 280-306 
Schmitt M, Knopp G, Materny A and Kiefer W (1998) The Application of Femtosecond Time-Resolved Coherent Anti-Stokes Raman Scattering for the Investigation of Ground and Excited State Molecular Dynamics of Molecules in the Gas Phase J Physical Chemistry A 102 4059-4065

Shkurinov A, Jonusauskas G and Rullière C (1994) Vibrational spectrum of liquid nitromethane revisited using polarization-sensitive coherent anti-stokes Raman scattering (PCARS) spectroscopy J Raman Spectroscopy 25 359-364
Tannor D J (2007) Introduction to Quantum Mechanics-A Time Dependent Perspective University Science Books California

Vierheilig A, Chen T, Waltner P, Kiefer W, Materny A and Zewail A H (1999) Femtosecond dynamics of ground-state vibrational motion and energy flow: polymers of diacetylene Chemical Physics Letters 312 349-356

Weiner A M (2011) Ultrafast optical pulse shaping: A tutorial review Optics Communication 284 3669-3692. 